M. Matsuda

Nagoya Math. J.

Vol. 103 (1986), 145-148

\title{
LIOUVILLIAN SOLUTIONS OF SECOND ORDER DIFFERENTIAL EQUATION WITHOUT FUCHSIAN SINGULARITIES
}

\author{
MICHIHIKO MATSUDA
}

\section{§. Introduction}

Consider a homogeneous linear differential equation of the second order whose coefficients are rational functions of the independent variable $x$ over the field $\boldsymbol{C}$ of complex numbers. We assume that the coefficient of the first order derivative vanishes:

$$
y^{\prime \prime}+s(x) y=0, \quad s \in C(x), \quad s^{\prime} \neq 0 .
$$

Theorem. Suppose that none of the singular points is Fuchsian. Then it is reducible over $C(x)$ if it has a liouvillian solution.

The condition that none of the singular points is Fuchsian can not be removed. The assumption that the coefficient of the first order derivative vanishes can not also be removed (cf. §2).

Our proof is based on the discussions of Kaplansky's book [2] in Picard-Vessiot theory. The coefficient field $C$ can be replaced by any algebraically closed field of constants of characteristic 0 . The existence of Picard-Vessiot extension was proved by Kolchin [3].

If $s(x)$ is a polynomial the only singular point is the infinity and it is not Fuchsian. In this case our result was obtained by H. P. Rehm [6]. For liouvillian solutions of this equation we have several results due to R. R. Hailperin (formerly R. M. Roberts) [1] and H. P. Rehm himself.

\section{$\S 1$. Proof of Theorem}

The infinity is a singular point, since the coefficient of the first order derivative vanishes. It is not Fuchsian by our assumption. Hence, if the equation has an algebraic solution it is reducible over $\boldsymbol{C}(x)$ because the conjugate elements over $C(x)$ are solutions of our equation. Therefore, we may assume that there is no algebraic solution. Suppose that our equation,

Received May 7, 1985. 
having a liouvillian solution, is irreducible over $C(x)$. Then there is a rational function $a(x)$ over $C$ which satisfies

$$
a^{\prime \prime}=3 a a^{\prime}+2 s^{\prime}-4 a s-a^{3} .
$$

This is due to Kaplansky [2, §25]. It takes the form:

$$
a(x)=\Sigma \frac{e}{x-c} \neq 0, \quad c, e \in C, \quad(e \neq 0)
$$

due to Liouville [4] (cf. M. Matsuda [5, §13]). Let us express $s(x)$ as the sum of partial fractions over $C$ :

$$
s(x)=P(x)+\Sigma \frac{e^{\prime}}{x-c^{\prime}}+\Sigma \frac{e^{\prime \prime}}{\left(x-c^{\prime}\right)^{2}}+\cdots, \quad P \in C[x] .
$$

Then either $P \neq 0$ or $P=0$ and $\Sigma e^{\prime} \neq 0$, since the infinity is not Fuchsian. First suppose that $c$ is not a singular point, that is $c \neq c^{\prime}$ for any $c^{\prime}$. Comparing the coefficients of $(x-c)^{-3}$ in (1) we have

$$
2 e=-3 e^{2}-e^{3},
$$

and $e$ is either -1 or -2 . Secondly suppose that $c$ is a singular point, that is $c=c^{\prime}$ for some $c^{\prime}$. Since it is not Fuchsian, there is a term in (2):

$$
\frac{g}{(x-c)^{n}}, \quad g=e^{(n)} \neq 0, \quad n>2
$$

such that

$$
e^{(j)}=0, \quad j>n .
$$

Comparing the coefficients of $(x-c)^{-n-1}$ in (1) we have

$$
0=g(-2 n-4 e) \text {, }
$$

and $e=-n / 2$. If $P \neq 0$ we indicate $\operatorname{deg} P$ and its leading coefficient by $m$ and $b$ respectively. Let us multiply both sides of (1) by $x^{1-m}$ and set $x=\infty$. Then we have

$$
0=b(2 m-4 \Sigma e)
$$

but it is impossible because each $e$ is negative. If $P=0$ we indicate $\Sigma e^{\prime}$ by $A$, which does not vanish. Let us multiply both sides of (1) by $x^{2}$ and set $x=\infty$. Then we have

$$
0=A(-2-4 \Sigma e)
$$


but it is impossible because each $e$ is not greater than -1 .

\section{§2. Counter example}

Consider a differential equation:

$$
x^{2} y^{\prime \prime}-\left[x^{n}+\frac{1}{4}\left(\frac{n^{2}}{4}-1\right)\right] y=0,
$$

where $n$ is an odd natural number. The origin is a Fuchsian singular point and the infinity is not Fuchsian. We have a liouvillian solution:

$$
y=x^{(1-n / 2) / 2} \exp \left( \pm \frac{2}{n} x^{n / 2}\right)
$$

We shall show that the equation is irreducible over $C(x)$. To the contrary suppose that it is reducible. Then there is a rational function $v(x)$ over $C$ which satisfies

$$
v^{\prime}+v^{2}=-s=x^{-2}\left[x^{n}+\frac{1}{4}\left(\frac{n^{2}}{4}-1\right)\right] .
$$

It takes the form:

$$
v=R+\frac{Q^{\prime}}{Q}+\frac{d}{x}, \quad Q, R \in C[x], \quad d \in C,
$$

since at any pole $c$ of $v(x)$ distinct from 0 and $\infty$ it is expressed as

$$
v(x)=\frac{1}{x-c}+t(x)
$$

such that $c$ is not a pole of $t(x)$. We have

$$
v^{\prime}+v^{2}=R^{2}+R^{\prime}+\frac{Q^{\prime \prime}}{Q}+\frac{2 R Q^{\prime}}{Q}+\frac{2 d}{x}\left(R+\frac{Q^{\prime}}{Q}\right)+\frac{d^{2}-d}{x^{2}} .
$$

The order of infinity is even and non-negative if $R \neq 0$, and less than -1 if $R=0$. This contradicts our assumption that $n$ is odd.

Consider a differential equation:

$$
x^{2} z^{\prime \prime}+z^{\prime}-\left[x^{n}+\frac{1}{4}\left(\frac{n^{2}}{4}-1\right)-\frac{1}{4} \frac{1}{x^{2}}+\frac{1}{x}\right] z=0,
$$

where $n$ is an odd natural number. The origin and the infinity are the only singular points and they are not Fuchsian. If we change the 
dependent variable $z$ into $y$ by

$$
z=y \exp \frac{1}{2 x}
$$

the above equation is obtained for $y$. Hence, there is a liouvillian solution:

$$
z=x^{(1-n / 2) / 2} \exp \left(\frac{1}{2 x} \pm \frac{2}{n} x^{n / 2}\right)
$$

The equation for $z$ is irreducible, since

$$
\frac{z^{\prime}}{z}=-\frac{1}{2} x^{-2}+\frac{y^{\prime}}{y}
$$

by (3) and the equation for $y$ is irreducible.

\section{BIBLIOGRAPHY}

[1] R. R. Hailperin (formerly R. M. Roberts), On the solvability of a second order linear homogeneous differential equation, Doctoral Dissertation, Univ. Pennsylvania, 1960.

[2] I. Kaplansky, An introduction to differential algebra, Hermann, Paris, 1957.

[ 3 ] E. R. Kolchin, Existence theorems connected with the Picard-Vessiot theory of homogeneous linear ordinary differential equations, Bull. Amer. Math. Soc., 54 (1948), 927-932.

[4] J. Liouville, Mémoire sur l'intégration d'une classe d'équations differentielles du second ordre en quantités finies explicites, J. Math. Pures Appl., 4 (1839), 423-456.

[5] M. Matsuda, Lectures on algebraic solutions of hypergeometric differential equations, Lectures Math., Dept. Math. Kyoto Univ., 15, Kinokuniya, Tokyo, 1985.

[6] H. P. Rehm, Galois groups and elementary solutions of some linear differential equations, J. Reine Angew. Math., 307 (1979), 1-7.

Yamadacho $27-6$

Kitashirakawa

Kyoto 606, Japan 